Artículo de Investigación E08A06 - Proyecto "Modelo de altas prácticas de Recursos Humanos en el mercado maquilador”. Universidad Autónoma de Tamaulipas. * Recibido: 28-01-2016. ^ Aprobado versión final: 02-03-2017 JEL: M31, M51, L3. ₹ Pp. 137-156

\title{
Impacto de la satisfacción en la generación de compromiso laboral en trabajadores de la maquila mejicana.
}

\section{Impact of satisfaction in the generation of labor commitment in mexican maquila workers.}

\author{
Mónica Lorena Sánchez Limón - Mariana Zerón Félix \\ Yesenia Sánchez Tovar
}

MÉJICO

\begin{abstract}
Resumen: Se analizó la influencia que ejerce la satisfacción de los empleados en el compromiso de los trabajadores en el mercado laboral de la maquila mexicana. Para ello se utilizó una muestra de 100 trabajadores de una maquiladora de Ciudad Victoria, en Tamaulipas, México; los datos se analizaron mediante ecuaciones estructurales. Los resultados indicaron que la satisfacción influye positiva y significativamente en el compromiso laboral. De esta manera se logró obtener evidencia empírica que relaciona estas dos variables en el mercado laboral maquilador. Un hallazgo fue que los factores extrínsecos de Herzberg, Mausner y Snyderman (1959), en oposición a lo planteado por ellos, poseen la capacidad de motivar a los empleados.
\end{abstract}

Palabras claves: satisfacción laboral; compromiso laboral; mercado laboral; maquila.

Abstract: The influence exerted by employee satisfaction on workers' commitment to the Mexican maquila labor market was analyzed. For this purpose, a sample of 100 workers from a maquiladora of Ciudad Victoria in Tamaulipas was used; the data were analyzed by structural equations. The results indicated that the satisfaction influences positively and significantly on the labor commitment. In this way it was possible to obtain empirical evidence that relates these two variables in the labor market of maquila. One finding was that the extrinsic factors of Herzberg, Mausner and Snyderman (1959) in opposition to what was proposed have the capacity to motivate employees.

Keywords: job satisfaction; laboral commitment; labor market; maquila.

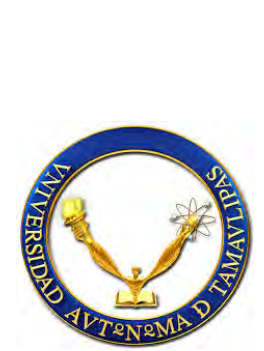

Mónica Lorena Sánchez es Doctora en Ciencias de la Administración por la UNAM, Maestra en Administración por la Universidad Autónoma de Tamaulipas, Licenciada en Computación Administrativa, profesora investigadora Universidad Autónoma de Tamaulipas. Contacto: msanchel@docentes.uat.edu.mx.

Mariana Zerón es Doctora en Administración, Maestra en Finanzas, Licenciada en Contaduría Pública, profesora investigadora, Grupo de investigación: Cuerpo académico Estrategias para el Desarrollo Regional. Institución: Universidad Autónoma de Tamaulipas.Contacto: zfmariana@uat.edu.mx.

Yesenia Sánchez es Doctora en Economía y Gestión de las Organizaciones por la Universidad de Zaragoza, España, Maestra en Economía, Licenciada en Contaduría Pública, profesora investigadora. Institución: Universidad Autónoma de Tamaulipas. Contacto: yesanchez@uat.edu.mx. 


\title{
Impacto da satisfação no compromisso de trabalho que gera entre trabalhadores mexicanos maquila.
}

\begin{abstract}
Resumo: A influência da satisfação do empregado o compromisso dos trabalhadores no mercado de trabalho da maquila mexicana analisados. Para isso, foi utilizada uma amostra de 100 trabalhadores de uma maquiladora em Ciudad Victoria, em Tamaulipas; os dados foram analisados por meio de equações estruturais. Os resultados indicaram que a satisfação positiva e significativamente influencia o compromisso de trabalho. Desta forma, foi possível obter evidência empírica que liga estas duas variáveis no mercado de trabalho maquiladora. Uma constatação foi a de que fatores extrínsecos Herzberg, Mausner e Snyderman (1959), em oposição às questões levantadas por eles, têm a capacidade de motivar os funcionários.
\end{abstract}

Palavras-chave: satisfação no trabalho; autorizações de trabalho; mercado de trabalho; maquila.

\section{Introducción}

1 n las últimas décadas las organizaciones han prestado mayor interés en la satisfacción de los empleados al reconocer que el disponer de trabajadores satisfechos representa un factor clave para mejorar el desempeño organizacional y, por el contrario, contar con empleados insatisfechos representa un verdadero reto para lograr niveles propicios de productividad (Meliá y Peiró, 1989; Abrajan y Contreras, 2009; Alonso, 2006; Martínez, 2007; García y Brás, 2008).

El contexto anterior ha ocasionado que la literatura también brinde un mayor nivel de relevancia a la satisfacción laboral en diversas áreas del conocimiento como la psicología, la economía y, principalmente, la referente a la dirección de empresas (Sterwart, 1996), por lo cual hoy en día es posible encontrar una gran cantidad de trabajos avocados al estudio de la satisfacción en relación con diversas variables como el clima organizacional, la productividad, la rotación del personal y el compromiso de los empleados con la organización (Bayona, Goñi y Madorran, 2000; Sanz, 2001; Villalba, 2001; García, Peiró, J.M. y Soro, 2003; Gamero Burón, 2004;. Sepúlveda y Segura, 2009; Zurita, Ramírez, Quesada, Ruiz, y Manzano, 2014).

De acuerdo con García, Peiró y Soro (2003), identificar los elementos que inciden en forma positiva o negativa en el nivel de satisfacción laboral es una actividad crucial para todas las empresas, al posicionarse la misma como un elemento con potencial para impulsar su desempeño. En este sentido, el presente trabajo tiene como objetivo analizar la relación existente entre la satisfacción de los empleados y el compromiso laboral. 


\section{La satisfacción laboral y su importancia}

En el entorno económico actual, caracterizado por ser sumamente complejo, dinámico y competitivo, las empresas se ven en la necesidad de dedicar mayores esfuerzos a la búsqueda de elementos que dispongan del potencial estratégico necesario que las ayuden a mejorar su desempeño organizacional e incrementar su participación en el mercado (Rodríguez, Retamal, Lizana y Cornejo, 2011). En este sentido, se reconoce que tanto los activos tangibles como los activos intangibles de las organizaciones poseen gran valor para el mejoramiento de la competitividad de las mismas.

Bori, Crespi y Mascarill (2012) sostienen que las empresas deben prestar igual atención tanto a los recursos materiales como a los no materiales de los que dispongan, y destacan a estos últimos como el verdadero elemento diferenciador entre las organizaciones. Al respecto, Álvarez (2004) y Atalaya (1999) indican que las actitudes, conocimientos, habilidades, experiencia y demás características individuales de los empleados son especialmente importantes para el eficiente desempeño de las organizaciones.

En este sentido, diversos autores señalan a la satisfacción laboral como uno de los aspectos propios de los individuos que más influencia tiene en su comportamiento y, subsecuentemente, en los resultados empresariales (Gattiker, y Larwood, 1988; Agho, Mueller y Price, 1993; Carmona y Leal, 1998), por lo que tal elemento ha sido estudiado y definido desde distintas perspectivas (Herzberg, Mausner y Snyderman, 1959; Davis y Newstrom, 1999; García, Peiró y Soro, 2003).

Herzberg, Mausner y Snyderman (1959) señalan que la satisfacción del empleado se refiere a la actitud del mismo, la cual refleja su sentimiento respecto al rol que desempeña en la organización. Para Davis y Newstrom (1999), la satisfacción laboral es la actitud asumida por un individuo en términos laborales, de la cual se espera que un empleado satisfecho muestre actitudes positivas laboralmente $y$, en caso contrario, un empleado insatisfecho reflejará actitudes negativas respecto a su trabajo.

Una definición muy amplia sobre el término es la propuesta por García, Peiró y Soro (2003: p.424), quienes indican que la satisfacción laboral se puede concebir como "una actitud global de carácter más o menos positivo ante diferentes aspectos de la experiencia laboral. Esa actitud implica una apreciación de la situación del trabajo en cada aspecto considerado y del grado en que esa situación responde a las expectativas y aspiraciones". 
De tal forma, se puede decir que la satisfacción laboral representa una respuesta emocional al puesto, ya sea positiva o negativa, y que se deriva de la evaluación entre las expectativas laborales del empleado y lo que realmente dicho puesto le permite obtener (Locke, 1976). En este marco es posible identificar dos elementos propios de la satisfacción del personal: en primera instancia la existencia de una actitud o sentimiento que se presenta hacia el trabajo y, en segundo lugar, que la misma es producto de una comparación entre las expectativas del puesto y las prestaciones que este ofrece realmente (Rodríguez, et al., 2011; Sánchez, 2008).

De acuerdo con Locke (1976), el concepto opuesto a la satisfacción en los empleados sería la insatisfacción laboral, debido a que este último elemento implica una actitud del personal de índole negativa en referencia con el puesto, y que a través de la cual se expresa frustración, negación de los valores laborales, falta de compromiso y un nivel bajo de desempeño.

Así, la satisfacción laboral se articula como un rubro de gran relevancia para las empresas, debido a que, si no existe un equilibrio entre lo que los empleados esperan de su puesto y lo que logran obtener del mismo, tal situación puede derivar en una actitud negativa hacia el trabajo, que de forma paralela pueda suponer un bajo rendimiento laboral (Locke, 1976; Rodríguez, et al., 2011).

Por lo anterior, es tarea de los directivos identificar los determinantes de la satisfacción o insatisfacción laboral dentro de las organizaciones. Sepúlveda y Segura (2009) y Bori, Crespi y Mascarilla (2012) postulan que el nivel de la satisfacción de los empleados se explica en gran medida por tres tipos de elementos. En primer lugar, se señala que la variabilidad en la satisfacción de los empleados puede explicarse por sus variables de personalidad, entre las que encuentran la estabilidad emocional y el grado de tolerancia al estrés.

El segundo arquetipo de elementos que inciden en la satisfacción laboral son los referentes a las características propias del trabajo, en las cuales se incluyen el salario, el horario de trabajo, las posibilidades de desarrollo profesional como el ascenso y las prestaciones. En tercer lugar, se establece una conjugación de los dos aspectos anteriores y en el que, además, se considera la apreciación personal que los empleados otorgan a dichas variables (Sepúlveda y Segura, 2009; Bori, Crespi y Mascarilla, 2012).

Para Alonso (2006), las condiciones de trabajo son los factores que explican en mayor medida la satisfacción en el personal de las empresas y señala (además del salario, la jornada laboral, las posibilidades de promoción) a las condiciones físicas del área de trabajo y a las relaciones personales que 
el puesto obligue o permita desarrollar, como aspectos que influyen para lograr o no un cumplimiento de las expectativas laborales del recurso humano de las empresas.

Sánchez (2008), por su parte, asevera que las particularidades del empleado, como la edad, el género y el nivel educativo, están asociadas de forma significativa con su nivel de bienestar laboral, pues tales características demostraron tener incidencia en los valores o prioridades del personal en relación con su trabajo. Al respecto, Clark (1997) y Sanz, (2001) mencionan que al momento de analizar o medir la satisfacción laboral, los elementos de mayor preponderancia son los rasgos del recurso humano.

Sánchez, Fuentes y Artacho (2007) destacan las circunstancias del entorno no laboral como elementos que también influyen en el cumplimiento de las expectativas laborales. Estos autores propiamente puntualizan el rubro familiar, pues indican que condiciones como los valores, el nivel de integración y el estilo de vida de dicho grupo social impactan de forma considerable en la actitud de los empleados con su trabajo y, por ende, lo que de él esperan.

Indubitablemente existe una gran cantidad de determinantes de la satisfacción laboral, sin embargo, es preciso mencionar que los elementos que explican la motivación de algunas personas pueden no influir en el cumplimiento de las expectativas laborales de otras o viceversa (Rodríguez, et al., 2011). Lo anterior se debe a la influencia de la percepción personal del empleado al momento de comparar los resultados obtenidos contra los esperados en lo relativo al puesto de trabajo (San Martín, 2013; Zurita, et al., 2014).

\section{Teoría de los dos factores}

Es ostensible que la satisfacción laboral se posiciona como un tópico de gran envergadura para las organizaciones y su desempeño, por lo cual las posibles causas de que los empleados estén satisfechos o insatisfechos con su trabajo han sido ampliamente estudiadas y, a su vez, han derivado en diversas teorías explicativas, dentro de las cuales sobresale la propuesta por Herzberg (1959) conocida como la "Teoría de los dos factores" o "Teoría Bifactorial".

En este sentido, Herzberg (1959) estipula la existencia de dos factores explicativos de la satisfacción laboral. El primero de ellos son los denominados higiénicos, y que se distinguen porque su carencia o inexistencia producen niveles bajos de satisfacción en los miembros de una empresa, sin embargo, si su nivel es suficiente no producen satisfacción. 
El segundo tipo de factor son los motivadores, cuya existencia incrementa el grado de satisfacción laboral cuando su nivel es el esperado y que, en caso contrario, de ser insuficientes, no logran provocar insatisfacción (Herzberg, 1959; Davis y Newstrom, 1991; Bori, Crespi y Mascarilla, 2012).

La característica diferenciadora entre ambos factores estriba en que los primeros son extrínsecos al trabajo, mientras que los segundos son intrínsecos al mismo (Peiró, 1984). De acuerdo con Herzberg, Mausner y Snyderman (1959) los factores higiénicos únicamente son capaces de prevenir la insatisfacción laboral, pero no son determinantes de la satisfacción laboral ya que esta es explicada por los factores motivadores.

Respecto a los factores higiénicos o extrínsecos se identifican el aumento o disminución del salario, las políticas de la empresa, las condiciones ambientales, la seguridad en el trabajo, la introducción de ideas propias en el trabajo, así como la participación en las discusiones, las relaciones interpersonales y el status (Herzberg, Mausner y Snyderman, 1959; Agho, Mueller y Price, 1993). Así, por ejemplo, una mayor seguridad en el trabajo no producirá satisfacción laboral; por otro lado, las malas relaciones interpersonales sí producirán insatisfacción (Ver tabla 1).

Por su parte, entre los factores motivadores o intrínsecos se pueden mencionar el reconocimiento recibido por parte de los directivos o compañeros de trabajo, las promociones, la realización exitosa del trabajo, la naturaleza de la tarea, la asunción de la responsabilidad, la supervisión, la identificación y posibilidad de compaginar el trabajo con otras actividades (Herzberg, Mausner y Snyderman, 1959; Carmona y Leal, 1998). En este caso se establece que un aspecto como la promoción del empleado es capaz de producir una mayor satisfacción, y que cuando se cuenta con un trabajo rutinario no se producirá insatisfacción.

Tabla 1. Teoría bifactorial de Herzberg.

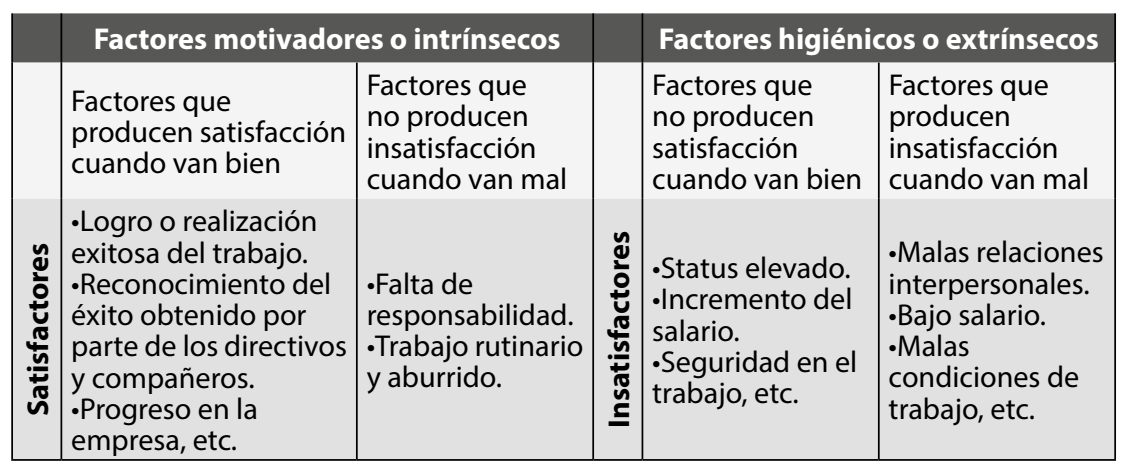

Fuente: Elaboración propia, basado en Herzberg, Mausner y Snyderman (1959). 
La teoría bifactorial establece que la satisfacción y la insatisfacción no necesariamente son dos vertientes totalmente opuestas como tradicionalmente se señala (Locke,1976), sino que en el caso de la satisfacción en las organizaciones lo contrario no es precisamente la insatisfacción, sino la ausencia de satisfacción y, en lo que corresponde a los empleados insatisfechos su lado opuesto no sería la satisfacción laboral, sino la ausencia de insatisfacción (Herzberg, Mausner y Snyderman, 1959; Davis y Newstrom, 1991).

\section{La satisfacción y compromiso laboral}

El compromiso organizacional se ha convertido en un elemento clave en la gestión de los recursos humanos, puesto que se asocia con el grado de lealtad o membresía que los empleados llegan a desarrollar hacia la organización (Álvarez de Mon et al., 2001), lo cual ha llevado a su estudio por parte de la teoría organizacional desde los años 70.

Para algunos autores el compromiso organizacional o laboral puede reflejar el grado en el cual el individuo se incorpora a la empresa y acepta y desarrolla las cualidades o valores que son aprobados por la organización (Gouldner, 1960), convirtiéndose de esta forma en un comportamiento deseable por parte de la administración. Esto lleva a los investigadores a tratar de definir cuáles elementos ayudan a manifestar este sentimiento de los empleados, surgiendo así la satisfacción laboral como un determinante de la misma.

La relación entre satisfacción laboral y compromiso ha sido punto central de diversos trabajos de investigación (Bayona, et al., 2000; Villalba, 2001; García y Brás, 2008; Gema, 2008; San Martín, 2013; Zurita, et al., 2014), mismos que coinciden en establecer que es de suma importancia para las empresas que su recurso humano se encuentre plenamente comprometido y satisfecho con su trabajo para la obtención de mejores resultados organizacionales.

Herzberg, Mausner y Snyderman (1959) sostienen que los empleados desempeñan un papel fundamental en las organizaciones, debido a que en ellos recae la responsabilidad de que la empresa realice sus actividades de forma eficiente y eficaz. No obstante, en la actualidad una de las problemáticas que enfrentan un gran número de empresas es lograr que sus empleados obtengan los niveles propicios de compromiso con su trabajo y, por ende, con las funciones inherentes al mismo (Gema, 2008).

Para García y Brás (2008) el compromiso organizacional fortalece el comportamiento responsable de los individuos, pues se fundamenta en los valores y objetivos de la organización. Según Bayona, et al. (2000), para la totalidad de organizaciones, sin importar tamaño o sector, es esencial 
que los individuos se encuentren adecuadamente comprometidos laboralmente, con el fin de poder alinear sus esfuerzos hacia el logro de los objetivos organizacionales.

Tradicionalmente se pensaba que el compromiso del recurso humano con su trabajo y con los objetivos de la organización estaba en función del aspecto financiero (Herzberg, Mausner y Snyderman, 1959). Sin embargo, hoy en día se estipula que la generación del compromiso organizacional va más allá, ello al identificarse que los empleados también tienen el interés de cubrir necesidades de otra índole con su trabajo, no solo económicas. (Gema, 2008; San Martín, 2013).

De tal forma que, en el ámbito empresarial moderno, las personas muestran mayores expectativas con relación a su trabajo, que además de brindarles estabilidad financiera también contribuyan a su bienestar y desarrollo personal (Villalba, 2001). Así, la satisfacción laboral es reconocida por la literatura como un factor explicativo del compromiso organizacional (Zurita, et al. 2014), pues la misma indica la percepción del individuo sobre el cumplimiento de los beneficios esperados de su trabajo, en comparación con los obtenidos realmente.

Por lo anterior se establece que los empleados más satisfechos también son los más comprometidos con la empresa, debido a que el compromiso mostrado por parte del trabajador con la organización se deriva del cumplimiento de las expectativas que este último tiene con su puesto (Villalba. 2001). En este mismo orden de ideas, García y Brás (2008) sostienen que la satisfacción laboral deriva en actitudes positivas en la vida laboral y privada.

Para Zurita, et al. (2014), en el momento en que una empresa contribuye a que su personal se sienta satisfecho con su trabajo, tanto en el contenido como con aspectos externos del mismo, de forma paralela logra incrementar el compromiso de sus empleados, quienes mostrarán un interés por colaborar en la consecución de los objetivos organizacionales. En contraparte, el recurso humano no satisfecho laboralmente no mostrará un empeño en la ejecución de sus funciones (Bayona, Goñi y Madorran, 2000).

Se propone explicar estadísticamente la influencia que ejerce la satisfacción laboral en el compromiso de los trabajadores dentro de la maquila mexicana. Se establece que el compromiso laboral está determinado por la satisfacción. Para verificar esta relación se realizó una prueba empírica con 100 trabajadores de una maquiladora, por medio de un análisis de regresión en SPSS, lo que aporta una evidencia sólida que fundamenta estadísticamente la veracidad predictiva de la relación propuesta.

Hipótesis 1: La satisfacción laboral influye positiva y significativamente en el compromiso de los empleados. 


\section{Metodología}

Con el fin de cumplir con el objetivo de investigación propuesto se consideró oportuno limitar el trabajo de campo a la empresa maquiladora local, en la cual se desarrolla una actividad laboral suficiente para garantizar resultados en la satisfacción laboral. Concretamente, la presente investigación se ha centrado en el estudio del conjunto de trabajadores operarios de la maquiladora Delphi Victoria II, de Ciudad Victoria, Tamaulipas, México.

En este sentido, esta investigación de corte cuantitativo es de tipo correlacional, causal y transversal. Puesto que el objetivo es determinar el impacto de la satisfacción laboral con el compromiso organizacional, se realizó, en primer lugar, un análisis factorial para comprobar los elementos que integran tanto la (SL) y el (CL); enseguida, un análisis de correlación entre las dimensiones resultantes; y, para finalizar, un análisis de regresión lineal multivariante. Para cumplir los objetivos previstos y contrastar la validez de la hipótesis planteada, se trabajó como se plantea en la figura 1.

Figura 1. Estructura de la investigación.

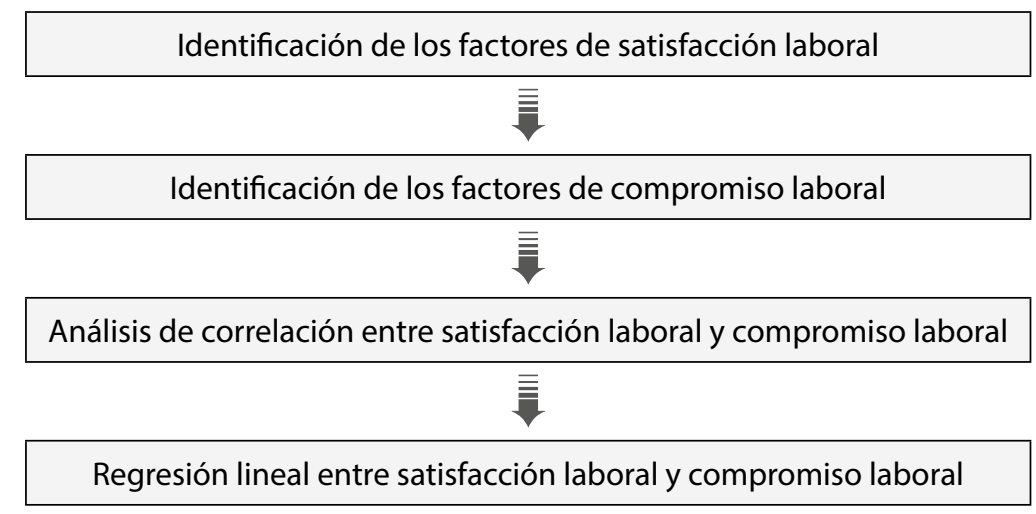

Fuente: Elaboración propia

\section{Variables del estudio}

Debido a que la hipótesis planteada es referida a la satisfacción laboral y el compromiso laboral, el estudio de dichos elementos se llevó a cabo a través de las variables que se presentan a continuación:

La satisfacción laboral, la cual se define como una actitud y sentimiento que el trabajador desarrolla hacia su puesto de trabajo y las condiciones del mismo, puede ser favorable o desfavorable, resultado de la percepción que desarrolla sobre sus distintas facetas o experiencias en el trabajo (Smith, Kendall, \& Hulin, 1969; Davis \& Newstrom, 1991; Robbins \& Judge, 2013). 
El compromiso laboral entre los participantes del sector maquilador es referido al vínculo de lealtad por el cual el empleado desea permanecer en la empresa (Álvarez de Mon et al., 2001).

\section{Participantes}

Con base en lo planteado, para este estudio la población está constituida por el conjunto de trabajadores operarios de una maquiladora de Ciudad Victoria, Tamaulipas; la muestra seleccionada fue por conveniencia, no aleatoria y no probabilística; se utilizó la fórmula de tamaño de muestra finita, ajustada a un nivel de confianza del $93 \%$ y un margen de error de $7 \%$; el tamaño de la muestra se estimó en 100 personas, no obstante se encuestaron 125 trabajadores, una vez eliminadas las encuestas con respuestas en blanco o aquellas que presentaban doble respuesta o respuesta confusa, la muestra definitiva fue de 100 personas.

Esta muestra final se compuso de 58 hombres y 42 mujeres, con edades comprendidas entre los 18 y los 59 años; en el caso de los hombres las edades presentaron una media de 27 años y una mediana de 26; las mujeres presentaron una media de 29 años y una mediana de 28 . Los trabajadores y trabajadoras pertenecen a la jornada matutina y vespertina de la empresa maquiladora. La antigüedad laboral fluctúa entre una semana y cinco años de labores.

\section{Medida de las variables}

\section{Satisfacción laboral (SL)}

No hay conceso en el uso de la escala más apropiada para medir la satisfacción laboral, mientras Suma \& Lesha (2013) señalan que la escala más apropiada y utilizada es la Job Descriptive Index (JDI) de Smith y otros (1969), otros autores como Llobet y Fito (2013) señalan que la escala más popular y apropiada para medir la satisfacción laboral es el Minnesota satisfaction questionnaire (MSQ). Para esta investigación, se utilizó una traducción y adaptación propia de la versión breve del MSQ; este nuevo cuestionario posee 10 ítems, medidos por cinco opciones tipo Likers, que van desde totalmente de acuerdo a totalmente en desacuerdo.

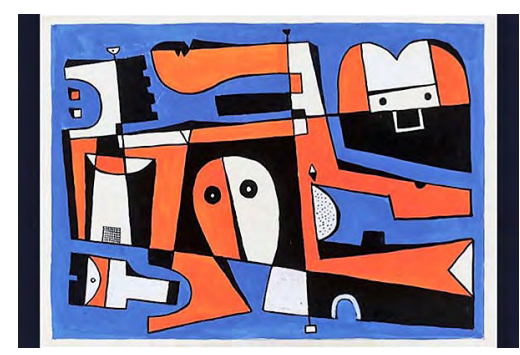


Tabla 2. Cuestionario traducido y adaptado de la MSQ versión breve.

1SL. Mi salario es adecuado y justo de acuerdo a las actividades que realizo en la empresa 2SL. Mi trabajo es seguro para mi salud

3SL. El servicio del comedor es agradable

4SL. Los servicios prestados por la enfermería son adecuados.

5SL. Estoy satisfecho con la supervisión de mi jefe

6SL. Los canales de comunicación son adecuados

7SL. Mis relaciones con mis compañeros de trabajo son satisfactorias

$8 \mathrm{SL}$. Mis supervisores me reconocen los méritos por mi trabajo o mis sugerencias

9SL. Se me da la oportunidad de crecer profesionalmente dentro de la empresa

10SL. Comparto los valores de la empresa

Fuente: Traducción y adaptación propia.

\section{Compromiso laboral (CL)}

La literatura proporciona varias escalas para medir el compromiso organizacional. En esta investigación la escala utilizada fue una traducción y adaptación propia basada en el organizational commitment questionnaire (OCQ) de Mowday, Steers y Porter (1979). Este nuevo cuestionario posee cinco ítems, medidos por medio de una escala Likert de cinco opciones que van desde totalmente de acuerdo a totalmente en desacuerdo.

Tabla 3. Escala para el Compromiso organizacional.

$1 \mathrm{CL}$. He pensado en la posibilidad de buscar otro trabajo o dedicarme a otra cosa 2CL. Mi asistencia al trabajo es excelente

$3 \mathrm{CL}$. Mi rendimiento laboral es el que la empresa me pide

$4 \mathrm{CL}$. Me siento orgulloso de pertenecer a esta empresa

$5 C L$. Estoy muy agradecido de trabajar en esta empresa

Fuente: Traducción y adaptación propia.

Tabla 4. Ficha Técnica de la investigación.

\begin{tabular}{|c|c|c|}
\hline \multirow{2}{*}{$\begin{array}{l}\text { Obtención de } \\
\text { la información }\end{array}$} & $\begin{array}{l}\text { Técnica seleccionada para la } \\
\text { obtención de la información }\end{array}$ & Encuesta personal \\
\hline & $\begin{array}{l}\text { Instrumento de obtención de la } \\
\text { información }\end{array}$ & $\begin{array}{l}\text { Cuestionarios } \\
\text { estructurados }\end{array}$ \\
\hline \multirow{5}{*}{ Muestreo } & Universo & $\begin{array}{l}\text { Personal de la maquiladora } \\
245 \text { empleados }\end{array}$ \\
\hline & Tamaño de la muestra & 100 empleados \\
\hline & Error & \pm 7 \\
\hline & Nivel de confianza & $93 \% p=q=50 \%$ \\
\hline & Diseño muestral & $\begin{array}{l}\text { Selección aleatoria de las } \\
\text { unidades muestrales }\end{array}$ \\
\hline \multirow{2}{*}{$\begin{array}{l}\text { Trabajo de } \\
\text { campo }\end{array}$} & Fecha de la aplicación de la técnica & Octubre 2015 \\
\hline & Lugar & Cd. Victoria, Tamaulipas \\
\hline
\end{tabular}

Fuente: Elaboración propia. 


\section{Procedimiento}

Para llevar a cabo la investigación se garantizó la confidencialidad de las respuestas a los participantes, sus identidades son anónimas; a cada uno se le entregó un cuestionario con 25 preguntas, diez de tipo categóricas y 15 de tipo cuantitativo. Las preguntas categóricas fueron de tipo sociodemográficas, como: edad, género, estado civil, dependientes económicos, antigüedad laboral, puesto de trabajo, entre otras. No obstante, las preguntas categóricas no forman parte de este análisis toda vez que no generaron diferencias significativas en la muestra estudiada. Las preguntas cuantitativas fueron 15 ítems: 10 de SL, versión traducida y adaptada de la Minnesota satisfaction questionnaire, y 5 ítems de compromiso laboral basado en el OCQ (Mowday, Steers y Porter, 1979).

\section{Resultados}

\section{Análisis factorial}

En primer lugar, se realizó un análisis de componentes principales para determinar los elementos que miden la satisfacción laboral y el compromiso laboral. Los indicadores de validez del método seleccionado han ofrecido resultados adecuados, por lo que la técnica utilizada se considera apropiada para explicar el comportamiento de las variables incluidas en el estudio. En la tabla 5 se pueden observar los indicadores de validez del método multivariante para la satisfacción laboral y el compromiso laboral.

Tabla 5. Indicadores de validez de los análisis factoriales de componentes principales.

\begin{tabular}{|l|c|c|}
\hline Índice Kaiser-Meyer-Olkin. & $\begin{array}{c}\text { Satisfacción } \\
\text { laboral }\end{array}$ & $\begin{array}{c}\text { Compromiso } \\
\text { laboral }\end{array}$ \\
Prueba de esfericidad de Bartlett (chi cuadrado) & .895 & .705 \\
Sig. & 373.889 & 219.715 \\
\hline
\end{tabular}

Fuente: Elaboración propia

Para determinar el número de factores a utilizar en la interpretación de los resultados se adoptó, en ambos casos, el criterio de conservar solo aquellos factores o dimensiones principales cuyos valores propios fueran mayores que la unidad. La tabla 6 muestra la varianza explicada para cada variable y los factores resultantes para la medición de las variables. 
Tabla 6. Factores identificados en el análisis factorial de componentes principales.

\begin{tabular}{|l|c|c|c|c|}
\hline & Factor & Total & \% de la varianza & \% acumulado \\
\hline \multirow{2}{*}{ Satisfacción laboral } & 1 & 5.067 & 50.670 & 50.670 \\
& 2 & 1.092 & 10.918 & 61.588 \\
Compromiso laboral & 1 & 2.855 & 71.385 & 71.385 \\
\hline
\end{tabular}

Fuente: Elaboración propia

Como se observa en la tabla anterior la satisfacción es explicada por dos factores, mientras que el compromiso laboral fue agrupado en una sola dimensión. Cabe destacar que los ítems agrupados en el primer factor de la satisfacción laboral corresponden en su totalidad a algunos que Herzberg, Mausner y Snyderman (1959) consideran como extrínsecos o higiénicos, mientras que los ítems que se agruparon en el segundo factor de la satisfacción laboral corresponden casi en su totalidad a los considerados como intrínsecos o motivacionales. Solo el ítem relacionado con la supervisión técnica se ubicó en el factor equivocado, esto posiblemente porque los entrevistados entendieron la pregunta más vinculada al reconocimiento que a la relación interpersonal.

Considerando que la muestra es igual a 100, se optó por eliminar aquellos ítems con cargas factoriales inferiores al 0.60 (Hair, et al., 2007). En la tabla 7 se exponen los elementos que conforman cada uno de los factores de la satisfacción laboral (SL).

Tabla 7. Matriz de componentes rotados de SL.

\begin{tabular}{|c|c|c|c|}
\hline \multirow{2}{*}{ Factor } & \multirow{2}{*}{ Ítem } & \multicolumn{2}{|c|}{ Componente } \\
\hline & & 1 & 2 \\
\hline \multirow{3}{*}{ Extrínseco } & $\begin{array}{l}\text { Mi salario es adecuado y justo, de acuerdo con las } \\
\text { actividades que realizo en la empresa }\end{array}$ & .050 & .895 \\
\hline & Mi trabajo es seguro para mi salud & .378 & .746 \\
\hline & $\begin{array}{l}\text { Los servicios prestados por la enfermería son } \\
\text { adecuados. }\end{array}$ & .551 & .561 \\
\hline \multirow{6}{*}{ Intrínseco } & El servicio del comedor es agradable & .610 & .314 \\
\hline & Estoy satisfecho con la supervisión de mi jefe & .828 & .099 \\
\hline & Los canales de comunicación son adecuados & .783 & .245 \\
\hline & $\begin{array}{l}\text { Mis supervisores me reconocen los méritos por mi } \\
\text { trabajo o mis sugerencias }\end{array}$ & .808 & .203 \\
\hline & $\begin{array}{l}\text { Se me da la oportunidad de crecer } \\
\text { profesionalmente dentro de la empresa }\end{array}$ & .790 & .232 \\
\hline & Comparto los valores de la empresa & .690 & .159 \\
\hline
\end{tabular}

Fuente: Elaboración propia 
Para comprobar la fiabilidad o consistencia interna de la escala se utilizó el alfa de Cronbach, la cual, de acuerdo con Hair et al. (2007), puede tener como límite inferior el .60.

En el caso de la satisfacción laboral dichos valores fueron aceptados, como se puede apreciar en la tabla 8. La tabla, además, muestra los descriptivos para cada factor del SL.

Tabla 8. Fiabilidad y descriptivos del SL.

\begin{tabular}{|c|c|c|c|c|}
\hline Factor & Ítem & Media & DS & $\mathbf{A}$ \\
\hline \multirow{3}{*}{ Extrínseco } & $\begin{array}{l}\text { Mi salario es adecuado y justo, de acuerdo } \\
\text { con las actividades que realizo en la } \\
\text { empresa }\end{array}$ & 3.075 & 1.367 & \multirow{3}{*}{0.740} \\
\hline & Mi trabajo es seguro para mi salud & 3.763 & 1.314 & \\
\hline & $\begin{array}{l}\text { Los servicios prestados por la enfermería } \\
\text { son adecuados. }\end{array}$ & 3.563 & 1.466 & \\
\hline \multirow{6}{*}{ Intrínseco } & El servicio del comedor es agradable & 3.750 & 1.445 & \multirow{6}{*}{0.878} \\
\hline & $\begin{array}{l}\text { Estoy satisfecho con la supervisión de mi } \\
\text { jefe }\end{array}$ & 3.613 & 1.571 & \\
\hline & $\begin{array}{l}\text { Los canales de comunicación son } \\
\text { adecuados }\end{array}$ & 3.688 & 1.346 & \\
\hline & $\begin{array}{l}\text { Mis supervisores me reconocen los méritos } \\
\text { por mi trabajo o mis sugerencias }\end{array}$ & 3.050 & 1.483 & \\
\hline & $\begin{array}{l}\text { Se me da la oportunidad de crecer } \\
\text { profesionalmente dentro de la empresa }\end{array}$ & 3.488 & 1.509 & \\
\hline & Comparto los valores de la empresa & 3.938 & 1.236 & \\
\hline
\end{tabular}

Fuente: Elaboración propia

Se eliminó un ítem con carga factorial inferior a 0.60 (Hair, et al. 2007). En la tabla 9 se exponen los elementos que forman el factor del compromiso laboral (CL).

Tabla 9. Matriz de componentes de CL.

\begin{tabular}{|c|l|c|}
\hline \multicolumn{1}{|c|}{ Factor } & \multicolumn{1}{|c|}{ Ítem } & \multicolumn{1}{|c|}{ Componente } \\
\hline $\begin{array}{l}\text { Compromiso } \\
\text { Laboral }\end{array}$ & $\begin{array}{l}\text { 2CL. Mi asistencia al trabajo es excelente } \\
\text { 3CL. Mi rendimiento laboral es el que la empresa } \\
\text { me pide } \\
\text { 4CL. Me siento orgulloso de pertenecer a esta } \\
\text { empresa } \\
\text { 5CL. Estoy muy agradecido de trabajar en esta } \\
\text { empresa }\end{array}$ & .819 \\
\hline
\end{tabular}

Fuente: Elaboración propia 
La consistencia interna de la escala del compromiso laboral resultó adecuada de acuerdo al alfa de Cronbach, con valores superiores al 0.6. La tabla 10 muestra los descriptivos para CL. La tabla, además, muestra los descriptivos para cada factor del CL.

Tabla 10. Fiabilidad y descriptivos del CL.

\begin{tabular}{|c|c|c|c|c|}
\hline Factor & Ítem & Media & DS & A \\
\hline \multirow{4}{*}{$\begin{array}{l}\text { Compromiso } \\
\text { Laboral }\end{array}$} & 2CL. Mi asistencia al trabajo es excelente & 3.43 & 1.26 & \multirow{4}{*}{0.864} \\
\hline & $\begin{array}{l}\text { 3CL. Mi rendimiento laboral es el que la } \\
\text { empresa me pide }\end{array}$ & 3.41 & 1.18 & \\
\hline & $\begin{array}{l}\text { 4CL. Me siento orgulloso de pertenecer a } \\
\text { esta empresa }\end{array}$ & 3.49 & 1.10 & \\
\hline & $\begin{array}{l}\text { 5CL. Estoy muy agradecido de trabajar en } \\
\text { esta empresa }\end{array}$ & 3.12 & 1.26 & \\
\hline
\end{tabular}

Fuente: Elaboración propia

\section{Análisis de correlación}

Para medir el grado de relación que guardan las dimensiones de la satisfacción laboral con el compromiso laboral, se calculó el coeficiente de correlación. Esto puede verse con mayor detalle en la tabla 11, donde se encontraron correlaciones de las dimensiones intrínseca y extrínseca de satisfacción laboral con la dimensión de compromiso laboral. Todas estas relaciones son positivas, cabe destacar que estas correlaciones guardan una alta significatividad de .01 .

Tabla 11. Correlaciones.

\begin{tabular}{|l|c|c|}
\hline \multicolumn{2}{|c|}{ Dimensiones de Satisfacción Laboral } & $\begin{array}{c}\text { Dimensión de } \\
\text { Compromiso Laboral }\end{array}$ \\
\hline Extrínseco & Correlación de Pearson & $.382^{* *}$ \\
Intrínseco & Sig. (bilateral) & .000 \\
& Correlación de Pearson & $.305^{* *}$ \\
\hline
\end{tabular}

** La correlación es significativa al nivel 0,01 (bilateral).

Fuente: Elaboración propia

\section{Análisis de regresión}

Dado el análisis de correlación anteriormente descrito al encontrar dos correlaciones significativas entre las dimensiones $\mathrm{SL}$ y $\mathrm{CL}$, se realizó un modelo estadístico de regresión lineal, en donde la variable dependiente 
fue compromiso laboral con las dimensiones de satisfacción laboral. Los resultados de esta estimación están en la tabla 12.

Tabla 12. Modelo estadístico de regresión del CL.

\begin{tabular}{|l|c|c|c|c|}
\hline \multirow{2}{*}{ Modelo } & \multicolumn{2}{|c|}{ Coeficientes no estandarizados } & \multirow{2}{*}{ Sig. } \\
\cline { 2 - 3 } & $\mathbf{B}$ & Error típ. & & \\
\hline (Constante) & $2.029 \mathrm{E}-17$ & .099 & .000 & 1.000 \\
SL Extrínseco & .382 & .099 & 3.844 & .000 \\
SL Intrínseco & .305 & .099 & 3.073 & .003 \\
& $\mathrm{~F}$ & 12.109 & & \\
Ajuste del modelo & $\mathrm{R}$ & 0.489 & \multirow{2}{*}{ Sig. $.000\left({ }^{* *}\right)$} \\
& $\mathrm{R} 2$ & 0.239 & & \\
& $\mathrm{R} 2 \mathrm{CORR}$ & 0.220 & \multicolumn{2}{|c}{} \\
\hline
\end{tabular}

* significatividad al nivel 0.01

Fuente: Elaboración propia

El coeficiente de determinación ajustado del modelo estadístico $\left(R^{2}\right)$ es 0.220 , lo que indica que dicho modelo explica la generación del compromiso laboral en un $22 \%$ aproximadamente, con una prueba F significativa de 12.109 ( $p$ valor .000), por lo que estos resultados corroboran la $\mathrm{H} 1$, que establece que la satisfacción laboral (en sus dos dimensiones) influye positiva y significativamente en el compromiso de los empleados.

\section{Conclusiones}

El propósito de esta investigación fue comprobar que la satisfacción laboral impacta de manera positiva en el compromiso laboral, donde se identificó que en el sector de la maquila el compromiso laboral está en función del grado de satisfacción de los empleados. De este modo, se resalta la relación significativa de la satisfacción laboral que afecta el nivel de compromiso con la empresa.

Esto apoya los resultados de Littlewod (2000), los cuales sugieren que las empresas que se esfuerzan en cubrir las necesidades de los empleados logran que sus empleados se comprometan con ellas, realizando sus actividades con factores como energia y afabilidad. Asi mismo apoya, en parte, la postura de Herzberg, Mausner y Snyderman (1959), quienes exponen en su trabajo que la motivación de los empleados está en relación con dos factores diferentes, uno extrínseco o higiénico y otro intrínseco o motivacional; no obstante lo anterior se oponen los resultados a estos autores en el sentido de que los factores extrínsecos poseen la capacidad 
de motivar a los empleados, toda vez que medidos como satisfactores fueron capaces de impactar en el compromiso laboral.

Los resultados deben ser interpretados con cautela, considerando que la muestra no representa a todo el universo de trabajadores de maquiladoras; sin embargo, este estudio tiene un gran valor al ser un estudio de caso estadísticamente confiable, en donde se logró obtener evidencia empírica que relaciona la satisfacción con el compromiso laboral en el mercado laboral maquilador.

La satisfacción es decisiva para elevar el compromiso laboral en el sector maquilador, en este sentido las empresas poco a poco van compenetrándose y entendiendo que la satisfacción del empleado es un factor vital para mejorar el compromiso laboral y las conductas derivadas de este (García y Brás, 2008; Gema, 2008; Zurita, et al., 2014) que incluyen la disminución del ausentismo (Somers, 1995), la reducción de la rotación laboral (Nazir, Shafi, Qun, Nazir, \& Tran, 2016; Payne \& Huffman, 2005) y el incremento del desempeño laboral (Meyer, Paunonen, Gellatly, Goffin, \& Jackson, 1989; De Cuyper \& De Witte, 2011).

\section{Referencias Bibliográficas}

- ABRAJAN, María y CONTRERAS, José Manuel. (2009, enero-junio). Grado de satisfacción laboral y condiciones de trabajo: una exploración cualitativa. En: Enseñanza e Investigación en Psicología, 14(1),105-118. Benito Juárez D.F: CNEIP.

- AGHO, Augustine O; MUELLER, Charles W; PRICE, James L. (1993). Determinants of employee job satisfaction: An empirical test of a causal model. En: Human Relations, 46(8), 1007-1027, California: Sage journals.

- $\quad$ ALONSO, Pilar. (2006, noviembre). Diferencias en la percepción de la satisfacción laboral en una muestra de personal de administración. En: Boletín de Psicología, 88, 49-63, Valencia: Universidad de Valencia.

- ÁlVAREZ, De Mon; CARDONA Santiago; CHINCHILLA Pablo; MILLER Nuria; PÉREZ, Paddy; PIN, Juan; Poelmans; RAMÓN, José. (2001). Paradigmas del Liderazgo. Madrid: McGraw Hill.

- ÁlVAREZ, Gema, (2008). Determinantes del compromiso organizacional. ¿Están los trabajadores a tiempo parcial menos comprometidos que los trabajadores a tiempo completo? En: Cuadernos de Estudios Empresariales, 18, 73-88. Madrid: Universidad Complutense.

- ÁlVAREZ, Gema., (2004). Análisis empírico de los determinantes de la satisfacción laboral en España. En: Revista de Economía y Empresa 52 y 53, 105-118. (2a época), 3.er cuatrimestre 2004 y 1.er cuatrimestre 2005. Academia Europea de Dirección y Economía de la empresa (AEDEM), España:Vigo.

- $\quad$ ATALAYA, María Clotilde (1999). Satisfacción laboral y productividad. En: Revista de la Facultad de Psicología de la Universidad Mayor de S. Marcos, III(5), 45-76. Lima: Universidad Mayor de S. Marcos. 
- $\quad$ BAYONA, Criatina., GOÑI, Salomé \& MODORRAN, Cristina (2000). Compromiso organizacional: implicaciones para la gestión estratégica de los Recursos Humanos. En: Revista Europea de Dirección y Economía de la Empresa, 9(1), 139149. Madrid: Elsevier.

- BORI, Sefa; CRESPI, Montserrat; MASCARILLA, Oscar. (2012): Variables determinantes de la satisfacción laboral en España. En: Cuadernos de Economía, 35, 9-16, Madrid: Elsevier.

- CARMONA, Antonio; LEAL, Antonio (1998). Teoría de los dos factores en la satisfacción del cliente. En: Investigaciones Europeas de Dirección y Economía de la Empresa, 4 (1), Pp. 53-80. Madrid: Elsevier.

- CLARK, Andrew. (1997). Job satisfaction and gender: why are women so happy at work? En: Labour Economics, vol. 4, pp. 341- 418. Madrid: Elsevier.

- DAVIS, Keith; NEWSTROM, John. (1999). Comportamientos Humano en el trabajo (10ed.). Mc Graw-Hill, México, D.F.

- DE CUYPER, Nele; DE WITTE, Hans. (2011). The management paradox: Self-rated employability and organizational commitment and performance. En: Personnel Review, 40(2), 152-172. Bingley: Emerald Group Publishing Limited.

- GAMERO, Carlos. (2004). Satisfacción laboral de los asalariados en España. En: Especial referencia a las diferencias por género. Cuadernos de Economía 27,109-146. Sevilla: Pablo D Olavide.

- GARCÍA, José; PEIRÓ, José; y SORO, Asunción. (2003). Capital Humano. Observatorio de la Inserción Laboral de los Jóvenes: 1996-2002. En: Instituto Valenciano de Investigaciones Económicas. Valencia: Bancaja.

- GARCÍA, Julio. y BRÁS, José. (2008). Satisfacción profesional y compromiso organizativo: un meta-análisis. En: Revista Europea de Dirección y Economía de la Empresa, 17(3), 61-78. Madrid: Elsevier.

- GATTIKER, Urs; LARWOOD, Laurie. (1988). Predictors for managers' career mobility, success, and satisfaction. En: Human Relations, 41, 569-591. California: Sage journals.

- GOULDNER, Helen. (1960). Dimensions of Organizational Commitment. En: Administrative Science Quarterly, 4(4), 569-591. California: Sage journals.

- HERZBERG, Frederick; MAUSNER, Bernard; SNYDERMAN, Bárbara. (1959): The motivation to work. En: Nueva York: John Wiley \& Sons. In Work and the nature of man, F. Herzberg (1973), New York: Mentor Book. Pp. 91-111.

- LITTLEWOOD, Herman. (2000), Compromiso organizacional: un estudio comparativo entre seis universidades, Proyecciones 6-1. En: División de Administración y Ciencias Sociales de la Rectoría Zona Sur, de El Instituto Tecnológico de Estudios Superiores de Monterrey (ITESM), Ciudad de México.

- LOCKE, Edwin A. (1976). The nature and causes of job satisfaction. In M.D. Dunnette (ed.). En: Handbook of Industrial and Organizational Psychology. Chicago: Rand McNally.

- LOCKE, Edwin A. (1984). Job satisfaction. En M. Gruneberg y T. Wall (eds.). En: Social Psychology and Organizational Behaviour. Chichester: Wiley.

- MARTínEZ, Noemí. (2007). Recursos humanos y management empresarial. El caso de la satisfacción laboral. En: Cuadernos de CC.EE. y EE., 52, 75-101. Málaga: Facultad de Ciencias Económicas y Empresariales, Universidad de Málaga. 
- MATHIEU, John E. y ZAJAC, Dennis M. (1990). A review and meta-analysis of the antecedents, correlates, and consequences of organizational commitment. En: Psychological Bulletin, 108, 171-194. Washington: American Psychological Association.

- MATHIEU, John; FARR, James. (1991). Further evidence for the discriminant validity of measures of organizational commitment, job involvement, and job satisfaction. En: Journal of Applied Psychology. Washington: American Psychological Association. Pp. 76, 127-133.

- MELIÁ, José; PEIRÓ, José. (1989). La medida de la satisfacción laboral en contextos organizacionales: El Cuestionario de Satisfacción S20/23. En: Psicologemas, 5, 5974. Valencia: Facultad de Psicología Universidad de Valencia.

- MEYER, John; PAUNONEN, Sampo; GELLATLY, lan; GOFFIN, Richard; JACKSON, Douglas. (1989). Organizational commitment and job performance: It's the nature of the commitment that counts. En: Journal of applied Psychology, 74(1),152-156. Washington: American Psychological Association.

- $\quad$ NAZIR, Sajjad; SHAFI, Amina; QUN, Wang; NAZIR, Nadia; TRAN, Quang. (2016). Influence of organizational rewards on organizational commitment and turnover. En: Employee Relations, 38(4), 596-619. Bingley: Emerald Group Publishing Limited.

- PAYNE, Stephanie; HUFFMAN, Ann. (2005). A longitudinal examination of the influence of mentoring on organizational commitment and turnover. En:Academy of Management Journal, 48(1), 158-168. New York: Academy of Management.

- PEIRÓ, José M. (1984). Psicología de la organización. Madrid: UNED.

- POZO, Carmen; MORILLEJO, Enrique; HERNÁNDEZ, Sonia; y MARTOS, María. (2005). Determinantes de la satisfacción laboral en trabajadores de la administración pública: el valor de las relaciones interpersonales en el lugar de trabajo. En: Ansiedad y Estrés, 11(2-3), 247-264. Madrid: Sociedad Española para el Estudio de la Ansiedad y el Estrés.

- RODRÍGUEZ, Andrés; RETAMAL, María;LIZANA, José;CORNEJO, Felipe (2011).Clima y Satisfacción laboral como predictores del desempeño: en una organización estatal chilena. En: Salud y Sociedad, 2(2), 247-264. Chile: Universidades Católica del Norte, Taica y Tarapacá.

- SAN MARTíN, Sonia (2013, abril-junio). La confianza, la satisfacción, las normas relacionales, el oportunismo y la dependencia como antecedentes del compromiso organizacional del trabajador. En: Contaduría y Administración 58(2),11-38. Ciudad de México: Universidad Nacional Autónoma de México.

- SÁNCHEZ, Carlos. (2008). Motivación, satisfacción y vinculación. ¿Es gestionable la voluntad de las personas en el trabajo? En: Acción Psicológica 5(1), 9-28. Madrid: Revistas UNED.

- SÁNCHEZ, Sandra; FUENTES, Fernando; ARTACHO, Carlos. (2007). La perspectiva de género en el análisis de la satisfacción laboral: una aplicación empírica mediante modelos logit y probit. En: Cuadernos de Gestión, 7(2), 55-67. Vizcaya: Universidad del País Vasco.

- SANZ, Anna (2001). Gender differences in job satisfaction and labour market participation: UK evidence form propensity score. Presentado en 13th EALE Annual Conference, 13-16 sept., Jyväskylä, Finlandia. 
- SEPÚLVEDA, Wilmer, SEGURA, Patricia (2009). Factores que afectan la satisfacción laboral de los profesionales en administración de empresas en Colombia. En Encuentro 2009 del Consejo Latinoamericano de Administración de Empresas CLADEA 2009, noviembre 7-9 de 2009. Guayaquil, Ecuador.

- $\quad$ SLOANE, Peter; WILLIAMS, Hector. (2000). Job satisfaction, comparison earnings and gender. En: Labour, 14, 473-502.Oxford: Fondazione Giacomo Brodolini and Blackwell Publishers.

- SOMERS, Mark. (1995). Organizational commitment, turnover and absenteeism: An examination of direct and interaction effects. En: Journal of Organizational Behavior, 16(1), 49-58. Nueva Jersey: John Wiley \& Sons, Ltd.

- VILLALBA, Olivia. (2001). Incremento de la satisfacción y del compromiso organizacional de los empleados a través del liderazgo efectivo. Academia. En: Revista Latinoamericana de Administración, 26, 5-17. Redalyc, Bogotá.

- ZURITA, Marta; RAMÍREZ, Teresa; QUESADA, Juan; RUIZ, Bernardo; MANZANO, Juan. (2014). Compromiso organizacional y satisfacción laboral en una muestra de trabajadores de los juzgados de Granada. En: REIDOCREA, 3, 17-25. Nueva Granada: Universidad de Granada.

\begin{tabular}{c|l}
\hline Para citar & $\begin{array}{l}\text { Sánchez, M.L.; Zerón, M. \& Sánchez, Y. (2017). Impacto de } \\
\text { la satisfacción en la generación de compromiso laboral en } \\
\text { este artículo: } \\
\text { trabajadores de la maquila mexicana. Teuken Bidikay Vol. } 8 \\
\text { N¹1.Pp. 137-156 }\end{array}$
\end{tabular}

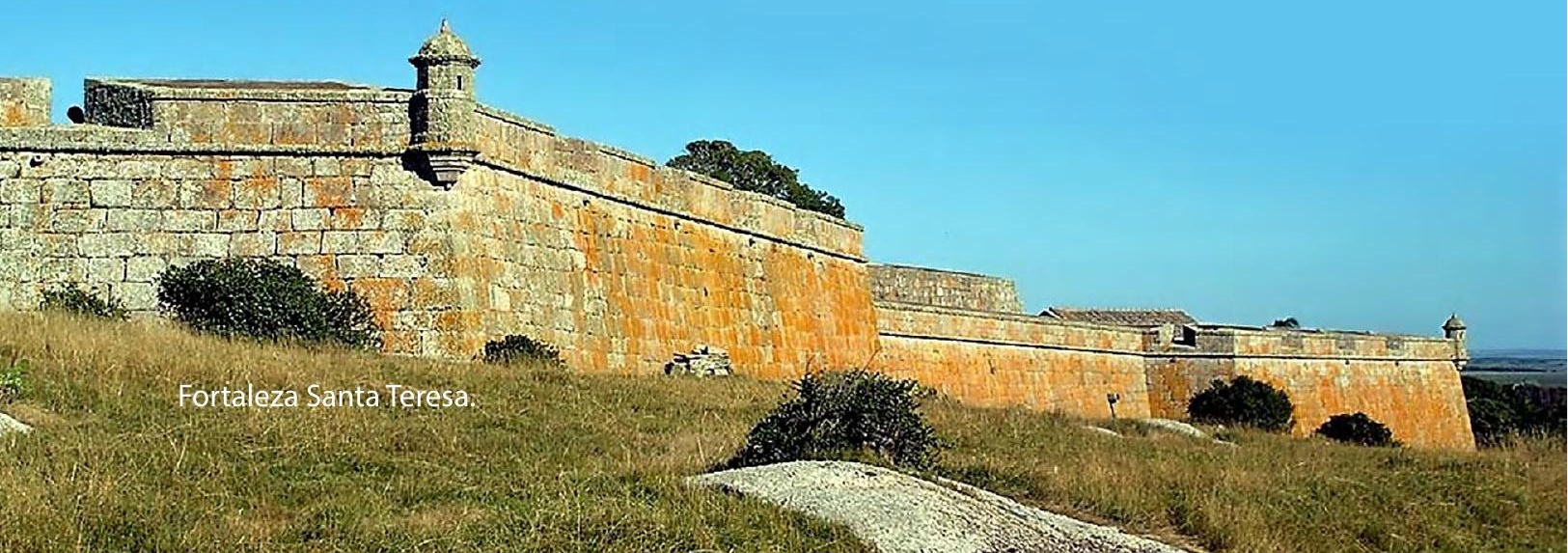

